\title{
Experimental investigation of flow through an asymmetric plane diffuser
}

\author{
By Carl U. Buice AND John K. Eaton ${ }^{1}$
}

\section{Motivation and objectives}

There is a need for experimental measurements in complex turbulent flows that originate from very well-defined initial conditions. Testing of large-eddy simulations and other higher-order computation schemes requires inlet boundary condition data that are not normally measured. The use of fully developed upstream conditions offers a solution to this dilemma in that the upstream conditions can be adequately computed at any level of sophistication. Unfortunately, experimenters have only recently been sensitized to this issue and there are relatively few appropriate data sets.

The plane diffuser experiment by Obi et al. (1993) has received a lot of attention because it has fully-developed inlet conditions and it includes separation from a smooth wall, subsequent reattachment, and redevelopment of the downstream boundary layer. Each of these features offers challenges for modern turbulence models. In particular, Durbin and Kaltenbach of CTR have devoted considerable effort in developing several different computations of the flow. Unfortunately, they found that the experiment had several deficiencies as they began careful comparison to the data. The most glaring problem is the fact that the data set does not appear to satisfy mass conservation, a problem that is most likely due to three-dimensional effects in the diffuser.

The objective of this study is to provide careful qualification and detailed measurements in a re-creation of the Obi experiment. The work will include extensive documentation of the flow two-dimensionality and detailed measurements required for testing of flow computations.

\section{Accomplishments}

The diffuser geometry as specified by Obi et al. is shown in Fig. 1. The expected flow includes flow separation approximately midway along the diffuser followed by reattachment in the tailpipe. The problem with this flow is that separation is likely to occur on the end-walls, causing an acceleration of the mid-plane flow. Our approach has been to modify an existing blower wind-tunnel to accommodate a very high aspect ratio version of the diffuser in hopes of minimizing end-wall effects. Unfortunately, the separated regions on the end-wall can be quite large and have a significant effect on the mid-plane flow. After construction, the majority of our efforts have been in controlling the end-wall boundary layer separation. 


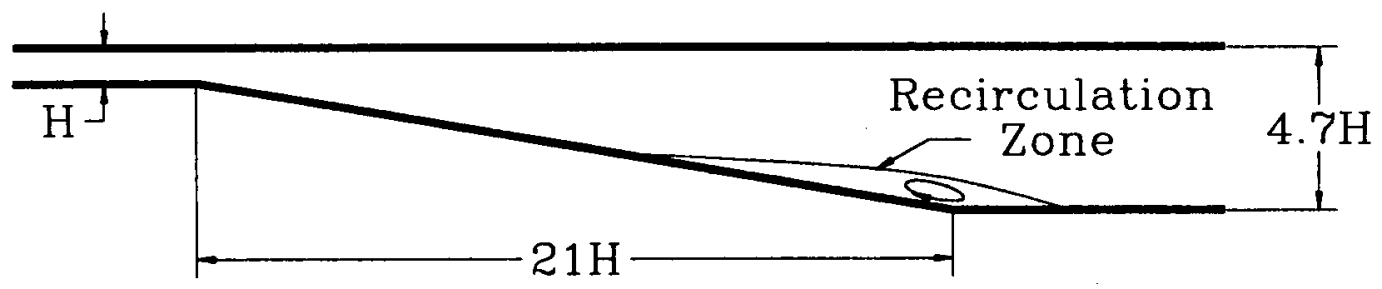

Figure 1. Plane diffuser.

\subsection{The experimental apparatus}

We have constructed an experimental facility, shown in Fig. 2, in the HTTM laboratory at Stanford. The facility has an upstream channel width $(H)$ of $1.5 \mathrm{~cm}$ and a depth of $60 \mathrm{~cm}$, giving an aspect ratio of 40 . The diffuser replicates the geometry of $\mathrm{Obi}$ et al., including the 10 degree asymmetric expansion to a total area ratio of 4.7. The blower tunnel can supply well-controlled flow sufficient to provide a channel Reynolds number $\left(U_{c l} H / \nu\right)$ ranging from approximately 10,000 to 30,000 . Two splitter plates have been installed $7 \mathrm{~cm}$ from the end-walls starting $6 H$ upstream of the beginning of the diffuser in order to remove the end-wall boundary layers. Holes have been drilled in the splitter plates within the first 5 channel heights of the diffuser to allow for the removal of the end-wall boundary layer through suction in the region of greatest adverse pressure gradient. Suction is developed by partial obstruction of the exit of the main section of the tunnel by a steel grid and by the presence of an adjustable obstruction located before the diffuser in the isolated end-wall sections.
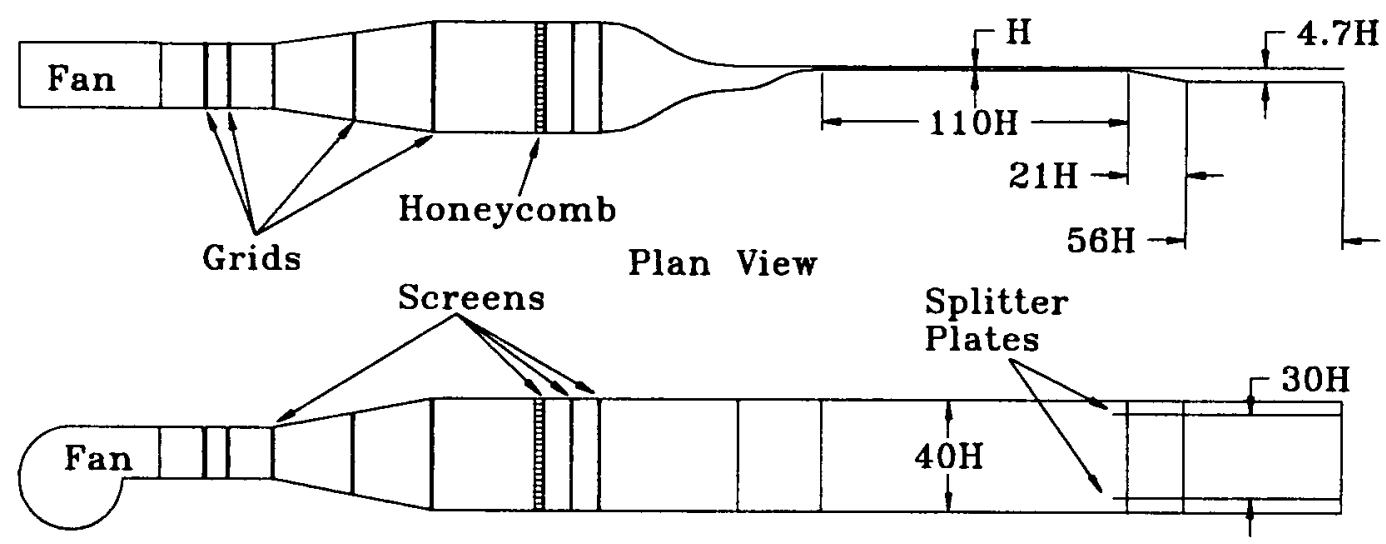

Side View

Figure 2. The experimental facility in HTTM.

\subsection{Preliminary results}

The first step in the qualification of the experimental facility included the removal of the diffuser section, leaving only the full development length of the channel. Wall pressure measurements and hot-wire mean velocity and turbulence profiles showed that the flow near the end of the channel was fully developed and spanwise 


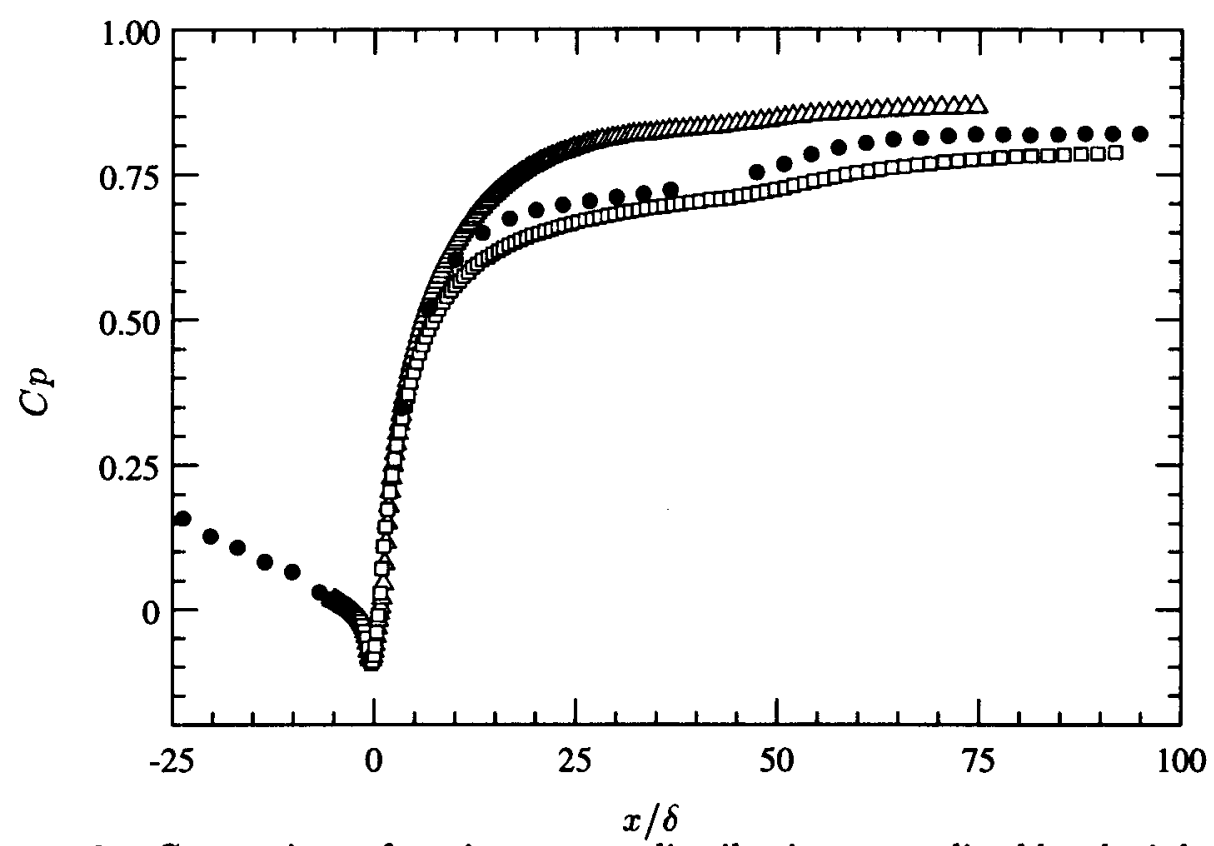

FIGURE 3. Comparison of static pressure distribution normalized by the inlet bulk velocity. Symbols: •, Experimental; $\Delta$, LES; $a, k-\varepsilon-\nu^{2}$.

homogeneous over $85 \%$ of the channel width. The streamwise pressure gradient was measured and verified against the value calculated from the velocity profile.

The diffuser section was then reinstalled and it became immediately obvious, through tuft flow visualization, that the end-wall boundary layers were severely separated in the outlet region of the diffuser. We choose splitter plates as the best approach for removal of the end-wall boundary layer that developed in the upstream channel flow. The splitter plates also provided a method for passive removal of the end-wall boundary layer in the diffuser. Using just the splitter plates, we were able to produce the correct pressure gradient in the inlet channel flow, which resulted in a pressure distribution that closely resembles the distribution calculated by Durbin, see Fig. 3. Unfortunately, integration of the velocity profiles upstream and downstream of the diffuser still showed a large discrepancy in the mass flow rates, indicating the continuing presence of secondary flow due to the influence of the end-wall region.

\section{Future work}

As soon as the tunnel qualification process is complete, hot-wire, wall pressure, and pressure probe measurements will be made in unseparated regions of the flow. These data will be augmented by thermal tuft measurements of the separation and reattachment locations. The final stage of the experiment will include taking detailed measurements of the flow using laser-Doppler anemometry for the velocity field measurements and pulsed-wall probes for the skin friction measurement. 


\section{REFERENCES}

KaltenBACH, H.-J. 1994 Large-eddy simulation of flow through a plane, asymmetric diffuser, Annual Research Briefs-1994, Center for Turbulence Research, NASA Ames/Stanford Univ. 175-184.

OBI, S., AOKI, K. \& MASUdA, S. 1993 Experimental and computational study of turbulent separating flow in an asymmetric plane diffuser; in: Ninth Symposium on Turbulent Shear Flows, Kyoto, Japan, August 16-19, 1993. p 305. 